\title{
Latent Autoimmune Diabetes in Adults (LADA) and its Metabolic Characteristics among Yemeni Type 2 Diabetes Mellitus Patients
}

\author{
Thekra Al-Zubairi \\ Molham AL-Habori $\mathbb{D}$ \\ Riyadh Saif-Ali
}

Department of Biochemistry and Molecular Biology, Faculty of Medicine and Health Sciences, University of Sana' a, Sana'a, Yemen
Correspondence: Molham AL-Habori Email malhabori@hotmail.com
Purpose: Although there is ample data about the prevalence of diabetes in the Middle East, little is known about the prevalence and features of autoimmune diabetes in this region. The aim of this study was to investigate the prevalence and metabolic characteristics of latent autoimmune diabetes in adults (LADA) amongst Yemeni Type 2 DM patients.

Patients and Methods: In this cross-section study, 270 Type 2 DM patients aged 30-70 years were recruited from the National Diabetes Center, Al-Thowra Hospital, Sana'a city, during the period November 2015 to August 2016. All Type 2 DM patients were diagnosed within 5 years and who did not require insulin for a minimum of 6 months following diagnosis. Levels of glutamic acid decarboxylase autoantibodies (GADA) were measured in all patients, and LADA was diagnosed in patients testing positive for anti-GAD antibodies. Further, biochemical analysis was carried out including fasting blood glucose (FBG), glycated haemoglobin $(\mathrm{HbAlc})$, insulin, and lipid profile. Insulin resistance (HOMA-IR) and $\beta$-cell function (HOMA- $\beta$ ) were calculated.

Results: The prevalence of LADA, as defined by GADA-positive, amongst patient with Type 2 DM was $4.4 \%$; with no significant difference in the prevalence between male $(5.8 \%)$ and female (3.4\%). LADA patients were younger than GADA-negative Type 2 DM. Body mass index, waist circumference, insulin and HOMA- $\beta$ were significantly lower in LADA patients, whereas triglyceride, cholesterol, HDL-c and HOMA-IR were non-significantly lower with respect to Type $2 \mathrm{DM}$. In contrast, FBG and HbAlc were significantly higher in LADA patients. Moreover, the prevalence of metabolic syndrome was significantly lower in LADA as compared with Type 2 DM. Only 2 out of the 12 GADA-positive (16.7\%) were on insulin treatment at the time of the study.

Conclusion: The prevalence of LADA in Yemeni Type 2 DM is lower than many of those reported in the literature, with no gender preference. Metabolic syndrome was significantly lower in LADA patients. Patients with LADA share insulin resistance with Type 2 DM but display a more severe defect in $\beta$-cell function, thus highlighting the importance of an early diagnosis of LADA, to correctly treat LADA patients, allowing safe and effective therapies. Keywords: latent autoimmune diabetes in adults, Type 2 DM, metabolic syndrome, insulin resistance, glutamic acid decarboxylase antibody

\section{Introduction}

Latent autoimmune diabetes in adults (LADA) is a hybrid form of diabetes ${ }^{1}$ that describes a subgroup of patients similar to Type 1 DM with markers of islet autoimmunity and shares features of Type $2 \mathrm{DM}$, including adult onset and insulin resistance. $^{2-5}$ It is probably the most prevalent form of autoimmune diabetes in 
general, ${ }^{6}$ and is characterized by genetic, phenotypic, and humoral heterogeneity, including different degrees of insulin resistance, high variability of the $\beta$-cell dysfunction and autoimmunity. $^{3,7-9}$ However, the major characteristics of LADA are mainly focused on 3 points: presence of islet autoantibodies, age at diagnosis of at least 30 years, and insulin independence for at least 6 months after diagnosis. $^{10,11}$ Epidemiological studies show that LADA may account for $2-12 \%$ of all cases of diabetes in adult population, which differs significantly in different ethnic groups. $^{12-15}$ Moreover, 4-14\% of patients diagnosed with Type 2 DM are positive for Type 1 DM associated autoantibodies which are diagnostic for LADA. ${ }^{4,5,12-18}$

Patients with LADA are at high risk of progression to insulin dependency. ${ }^{19}$ A proportion of subjects with adultonset autoimmune diabetes does not require insulintherapy at the time of diagnosis and are clinically similar to patients with Type 2 DM. Although LADA appears similar to Type 2 DM, the typical presence of islet autoantibodies, such as GADA and tyrosine phosphatase antibody (IA-2A), indicate an autoimmune basis of LADA with a delayed progression compared with classic Type 1 DM. $^{20}$ The titre of antibody positivity especially GADA as well as the recognition of different patterns of autoantibody positivity can influence the clinical features of LADA and may be useful for diabetes classification and prediction of risk for insulin therapy. ${ }^{12,14,16,21}$ Overall, LADA patients with high GADA titer resemble Type 1 DM more closely, and those with low GADA titer resembles Type $2 \mathrm{DM}$ patients in their metabolic characteristics and $\beta$-cell function during follow-up. ${ }^{2}$

The association of LADA with Type 1 DM and Type 2 DM still remains unclear and the precise definition of LADA has always been controversial., ${ }^{4,22}$ Striking heterogeneity has been recently highlighted as a relevant characteristic of LADA. ${ }^{3}$ Patients with LADA shares clinical and metabolic features with Type 1 and Type $2 \mathrm{DM}$ as part of a range of variable severity of immune and metabolic dysfunction, ${ }^{23}$ described as "end of the rainbow". 7 Latent autoimmune diabetes in adults has Type 1 DM-like autoimmune genetic components and Type 2 DM-like metabolic genetic components. When compared with classical Type 1 DM, LADA appears like the other extreme of the autoimmune diabetes spectrum, whereby genetic susceptibility, autoimmune response and non-insulin-necessity presentation constitute a mild form of autoimmune diabetes with pathological features closer to those of Type 2 DM than to those of Type $1 \mathrm{DM}^{3}$ The lack of optimal treatment can result in deterioration of the autoimmune process, acceleration of $\beta$-cell loss, faster progression to insulin dependence, and an increased risk of complications. $^{1,3}$ However, a personalized medicine approach is needed to achieve optimal metabolic control and preserve $\beta$-cell function, which is associated with a lower risk of occurrence of long-term diabetes complications.

In view of the fact that LADA represents an important clinical issue that needs to be addressed in order to facilitate improved glycemic control as well as the preservation of residual $\beta$-cell function together with its limited data on the prevalence and metabolic features in the Middle East region; the aim of this study was therefore to examine the prevalence and the metabolic characteristics of LADA in Yemeni Type 2 DM patients.

\section{Patients and Methods Study Design, Subjects and Data Collection}

In this cross-section study, 270 Type 2 DM patients aged 30-70 years were enrolled from the National Diabetes Center, Al-Thowra Hospital, Sana'a city, from November 2015 to August 2016. All the Type 2 DM patients were diagnosed more than 2 months and less than 5 years, and who did not require insulin for a minimum of 6 months following diagnosis. Patients with secondary diabetes mellitus due to other conditions or under immunosuppression, pregnant women, heavy ketonuria $(++)$ on urine test sticks, renal disease, or acute illness at the time of testing were excluded from this study. The study protocol was approved by the Institutional Review Board (IRB) of the Faculty of Medicine and Health Sciences, Sana'a University. Informed consent form was obtained from all individuals after explaining the purpose and nature of the study.

The patients' height and weight were measured and body mass index (BMI), defined as weight $(\mathrm{kg}) /$ height squared $\left(\mathrm{m}^{2}\right)$, was calculated. Waist circumference (WC) was measured halfway between the lower rib margin and the anterior superior iliac spine. Blood pressure (BP) was measured in the seated position using an Omron IntelliSense Automatic Blood Pressure Monitor with at least a $10 \mathrm{~min}$ rest before period before the measurement. Two to three successive BP readings were obtained at 5 min intervals and averaged. Standardized questionnaire(s) was administered to collect patients' demographic and 
clinical data including: age; age at onset of diabetes; duration of diabetes; family history of diabetes, the current treatment regimen that the patient is following; whether on insulin for glycaemic control; history of hypertension and dyslipidaemia.

Cases of LADA were defined as Type 2 diabetic subjects with diabetes-associated autoantibodies who did not develop ketoacidosis or did not require insulin for at least 6 months after diagnosis. ${ }^{11}$ Metabolic syndrome (MetS) was classified according to the harmonized metabolic syndrome criteria, ${ }^{24}$ whereby any 2 of the 4 following factors were required for the diagnosis of MetS among Type 2 DM patients: $\mathrm{WC}$ in $\operatorname{man} \geq 94 \mathrm{~cm}$ and women $\geq 80 \mathrm{~cm}$; triglyceride (TG) $\geq 150 \mathrm{mg} / \mathrm{dl}$; HDL-cholesterol (HDL-c) in $\mathrm{man}<40 \mathrm{mg} / \mathrm{dl}$ and women $<50 \mathrm{mg} / \mathrm{dl}$; systolic blood pressure $(\mathrm{SBP}) \geq 130 \mathrm{mmHg}$, and diastolic blood pressure $(\mathrm{DBP}) \geq 85 \mathrm{mmHg}$.

\section{Blood Collection and Biochemical Analysis}

A fasting venous blood $(5 \mathrm{~mL})$ was collected from each patient after an overnight fast of more than 10 hours and divided into two vacuumed tubes; $4 \mathrm{~mL}$ into plain tubes for biochemical assay and $1 \mathrm{~mL}$ into a $\mathrm{K}_{2}$ EDTA tube for glycated haemoglobin $(\mathrm{HbAlc})$. The serum from each sample was separated within 30 minutes and stored at $20^{\circ} \mathrm{C}$ for biochemical analysis. Haemolysate was prepared immediately for $\mathrm{HbAlc}$ determination within 2 hours of blood collection. Fasting blood glucose (FBG), TG, total cholesterol, and HDL-c were measured on an automated analyzer, the Cobas c501 (Roche Diagnostic, Germany), using their respective kits (Roche Diagnostic, Germany). Glycated haemoglobin (HbA1c) was measured on the Cobas c501 automated analyzer (Roche Diagnostic, Germany) using a turbidimetric inhibition immunoassay. Insulin was measured by an electrochemiluminescence immunoassay (ECL) on an Elecsys autoanalyzer (Roche Diagnostic, Germany). Insulin resistance (HOMA-IR) and $\beta$-cell function (HOMA- $\beta$ ) were calculated using the Homeostasis Model Assessment (HOMA2) Calculator v2.2 which is available from the Oxford Centre for Diabetes, Endocrinology, and Metabolism. Glutamic acid decarboxylase antibody (GADA) was measured using enzyme linked immunosorbent assays (Euroimmun AG, Luebeck, Germany), with the optimal cut-off value of positivity set at $10 \mathrm{U} / \mathrm{mL}$. The assay demonstrated good performance when tested in the 2015 Islet Autoantibody
Standardization Program (IASP) Workshops, with a sensitivity of $82 \%$ and specificity of $99 \%$.

\section{Statistical Analysis}

The statistical analyses were performed on Social Package of Social Sciences (SPSS) version 11.5 (SPSS Inc, Chicago, IL, USA). The diabetic and metabolic syndrome parameters were skewed and therefore normalized by logarithmic transformation. Means were subsequently back transformed for presentation as geometric means and $95 \%$ confidence interval. Independent $t$-test was used to compare the diabetic and metabolic syndrome parameters between LADA and Type 2 DM. The treatment and pattern of metabolic syndrome factors among Type 2 DM and LADA were evaluated by Chisquare test. The significant differences were indicated if p-value was $<0.05$.

\section{Results}

Based on the detection of positive glutamic acid decarboxylase autoantibodies (GADA) $\geq 10 \mathrm{IU} / \mathrm{mL}$, the prevalence of LADA among the 270 Type 2 DM recruited in this study was $4.4 \%$ (12 patients). Comparison of the demographic and biochemical parameters between GADA-negative Type 2 DM and LADA patients is depicted in Table 1 . Of the 12 diagnosed LADA patients, seven $(58.3 \%)$ were male and five $(41.7 \%)$ were female; with no significant difference between them. Both age and age of onset were significantly $\left(p=1 \times 10^{-5}, p=8 \times 10^{-5}\right)$ lower in LADA by $23 \%$ and $22 \%$ as compared with Type $2 \mathrm{DM}$. Moreover, BMI and WC were also significantly $\left(\mathrm{p}=1 \times 10^{-3}, \mathrm{p}=9 \times 10^{-3}\right)$ lower in the LADA group by $17.9 \%$ and $9.9 \%$, respectively. However, SBP and TG were non-significantly lower in the LADA group with respect to Type $2 \mathrm{DM}$ by $3.4 \%$ and $19.3 \%$; with no difference in DBP, cholesterol and HDL-c.

Fasting blood glucose and $\mathrm{HbA} 1 \mathrm{c}$ were significantly $(\mathrm{p}=1$ $\times 10^{-4}$ ) higher in LADA group by $56.5 \%$ and $34 \%$ as compared with Type 2 DM. On the other hand, insulin and HOMA- $\beta$ were significantly $\left(\mathrm{p}=1 \times 10^{-4}\right)$ lower in LADA group by $53 \%$ and $74.5 \%$, respectively; whereas HOMA-IR was non-significantly lower by $16.7 \%$. The use of oral hypoglycemia, hypertension, or hyperlipidemia drugs was nonsignificantly different between the two groups (Table 1), with only 2 LADA patients being on insulin treatment. Moreover, diabetic family history was also non-significantly different between LADA and Type 2 DM.

Table 2 shows metabolic syndrome to be significantly $(\mathrm{p}=0.041)$ lower in LADA patients $(41.7 \%)$ with respect to Type 2 DM (70.5\%). Similarly, increased WC was 
Table I Demographic and Clinical Characteristics of Both Type 2 DM (GADA-Negative) and LADA (GADA-Positive)

\begin{tabular}{|c|c|c|c|}
\hline & Type 2 DM $(n=258)$ & LADA $(n=12)$ & P value \\
\hline \multicolumn{4}{|l|}{ Gender } \\
\hline Male & II 4 (44.2\%) & 7 (58.3\%) & \\
\hline Female & I 44 (55.8\%) & $5(41.7 \%)$ & 0.252 \\
\hline Age (years) & $48(47-49)$ & $37(33-42)$ & $1 \times 10^{-5}$ \\
\hline Age at Onset (years) & $45(44-47)$ & $35(32-38)$ & $8 \times 10^{-5}$ \\
\hline BMI $\left(\mathrm{kg} / \mathrm{m}^{2}\right)$ & $28(27-29)$ & $23(20-26)$ & $1 \times 10^{-3}$ \\
\hline Waist circumference $(\mathrm{cm})$ & 91 (89-92) & $82(75-88)$ & $9 \times 10^{-3}$ \\
\hline $\mathrm{SBP}(\mathrm{mmHg})$ & $118(117-120)$ & $114(106-122)$ & 0.263 \\
\hline $\mathrm{DBP}(\mathrm{mmHg})$ & $81(80-83)$ & $8 \mid(75-87)$ & 0.86 \\
\hline Triglyceride (mg/dl) & $166(156-176)$ & $134(10 \mid-176)$ & 0.132 \\
\hline Cholesterol (mg/dl) & $178(172-185)$ & $170(146-200)$ & 0.591 \\
\hline HDL-c (mg/dl) & $54(52-57)$ & $52(43-64)$ & 0.729 \\
\hline FBS (mg/dl) & $168(160-176)$ & $263(211-327)$ & $1 \times 10^{-4}$ \\
\hline $\mathrm{HbAlc}(\%)$ & $8.5(8.2-8.7)$ & II.4 (9.8-13) & $1 \times 10^{-4}$ \\
\hline Insulin $(\mathrm{pmol} / \mathrm{l})$ & $116(107-126)$ & $54(37-79)$ & $1 \times 10^{-4}$ \\
\hline HOMA-IR & $2.4(2-3)$ & $2(I-3)$ & 0.416 \\
\hline HOMA- $\beta(\%)$ & $47(43-52)$ & $12(8-19)$ & $1 \times 10^{-4}$ \\
\hline Oral hypoglycemia drug & $231(89.5 \%)$ & $10(83.3 \%)$ & 0.376 \\
\hline Hypertension drug & $63(24.4 \%)$ & $2(16.7 \%)$ & 0.416 \\
\hline Hyperlipidemia drug & 45 (I7.4\%) & $2(16.7 \%)$ & 0.652 \\
\hline Diabetic family history & $156(60.5 \%)$ & $7(58.3 \%)$ & 0.553 \\
\hline
\end{tabular}

Notes: Data presented as geometric mean ( $95 \%$ Confidence interval). Mean significant difference $p<0.05$ bolded.

Table 2 Pattern of Metabolic Syndrome Factors Among Type 2 DM and LADA

\begin{tabular}{|l|c|c|c|}
\hline & Type 2 DM (n = 258) & LADA (n = 12) & P value \\
\hline Metabolic syndrome & $182(70.5 \%)$ & $5(41.7 \%)$ & $\mathbf{0 . 0 4 0}$ \\
Increased waist circumference & $167(64.7 \%)$ & $4(33.3 \%)$ & $\mathbf{0 . 0 3 1}$ \\
High blood pressure & $117(45.4 \%)$ & $4(33.3 \%)$ & 0.203 \\
High triglyceride & $159(61.6 \%)$ & $5(41.7 \%)$ & 0.14 \\
Decreased HDL-c & $103(39.9 \%)$ & $5(41.7 \%)$ & 0.498 \\
\hline
\end{tabular}

Notes: Data presented as numbers (\%). Significant difference $p<0.05$ bolded.

significantly $(\mathrm{p}=0.031)$ lower in LADA $(33.3 \%)$ as compared with Type 2 DM (64.7\%). However, increased BP and TG were non-significantly lower in LADA than Type 2 DM; with decreased HDL-c being non-significantly different between the two groups.

\section{Discussion}

The results presented in this study show the prevalence of LADA as defined by GADA positive amongst patient with Type $2 \mathrm{DM}$ was $4.4 \%$, which is similar to those reported in a Korean population study $(4.4 \%)^{25}$ and in a study from North America and Europe on drug-naive Type 2 DM patients $(4.2 \%) .{ }^{26}$ However, the prevalence in our study was higher than the study conducted in South Africa (2.5\%), ${ }^{27}$ United
Arab Emirates (2.6\%), ${ }^{12}$ Malaysia (2.9\%), ${ }^{28}$ and lower than those reported in Sardinia (5.1\%), ${ }^{29}$ Tanzania (5.3\%), ${ }^{30}$ Sri Lanka (5.4\%), ${ }^{31}$ China $(5.9 \%)^{14}$ and the Cuttack region of Eastern India (7\%). ${ }^{32}$ Higher prevalence was reported in studies done in Nigeria $(11.9-14 \%)^{33,34}$ and Ghana (13.5\%). ${ }^{35}$ The UK Prospective Diabetes Study (UKPDS), which is one of the largest studies done in patients with Type 2 DM, reported a LADA prevalence of $11.2 \%{ }^{36}$ Moreover, several European studies done in various groups of patients with clinically diagnosed as Type 2 DM in Finland, Australia, United Kingdom, and Sweden have found the frequency of GADA-positivity to range between 2 and $21 \%{ }^{3,37}$

The differences in prevalence from the various studies may be attributed to ethnic variation, differences in sample 
selection techniques, the use of different assay techniques for detection of GADA and varying diagnostic criteria or cut-off points. Moreover, differences in dietary habits, environmental factors, and phenotypic characteristics between European and Asian populations are reported to be responsible for the heterogeneity in the prevalence and characteristics of LADA in these 2 populations. ${ }^{19}$ In addition, population-based studies have reported lower prevalence of islet autoantibodies (including GADA) when compared with hospital-based studies. ${ }^{38,39}$ Currently, GADA is considered to be the most sensitive and specific biomarker in the diagnosis of LADA. ${ }^{5,40,41}$ Moreover, GADA was also demonstrated to be the most frequent islet autoantibody in both elderly and young LADA patients $^{42}$ and was proposed that detection of GADA autoantibody alone is adequate when screening for LADA patients. On the other hand, a previous study reported that since GADA disappear after prolonged disease duration, ${ }^{41}$ it is likely that some LADA patients therefore appeared GADA-negative and classified as having Type 2 DM.

Moreover, our results showed no significant difference in the prevalence between male (5.8\%) and female (3.4\%) suggesting that there is no gender preference for LADA which is supported by earlier reports. ${ }^{18,34}$ However, other studies reported higher proportion of GADA-positive males than females amongst Type 2 DM patients. ${ }^{43,44}$ Our study also showed that LADA patients are younger than GADA-negative Type 2 DM which is in agreement with an earlier study; ${ }^{28,36}$ but differ from another study reporting no change in age. ${ }^{26}$ One explanation could be that $\beta$-cell function declines rapidly in very young patients but slows in adolescence and adults. ${ }^{45}$ Alternatively, genetic influences were reported to be determinants of age at presentation in LADA specifically the DRBI/ DOBI genotype which is demonstrated in younger LADA patients. ${ }^{46}$ Moreover, the susceptibility of the elderly LADA ( $\geq 60$ years) was suggested to be is associated with a single HLA-DQ haplotype while that of the young LADA ( $<60$ years) is associated with multiple HLA-DQ haplotypes. ${ }^{42}$ Other studies recognized that the common variants in TCFL72 gene may differentiate in young but not middle-aged GADA-positive and GADAnegative diabetic patients. ${ }^{47}$ In addition, the higher prevalence of multiple autoantibodies in younger patients may be a possible cause for disease onset earlier in life. ${ }^{48}$ A recent study, however, showed no differences in age at diagnosis, duration of diabetes, or ethnic distribution was observed between GADA-negative and positive patients. ${ }^{49}$

Both BMI and WC of our LADA patients were significantly lower than Type $2 \mathrm{DM}$, which is in agreement with those reported for European and Asian populations. ${ }^{1,5,33,50-52}$ In addition, several studies have reported significantly lower BMI in GADA-positive patients, irrespective of the ethnicity, age or gender distribution of the population studied. ${ }^{36,43,44}$ Along the same line, another study showed BMI and WC, though not significant, to be lower in $\mathrm{LADA}^{33}$ and further suggested that there was a trend toward low values in GADA-positive individuals with Type 2 DM compared with the GADA-negative ones. A previous study reported that $76 \%$ of their LADA patients displayed BMI values corresponding to healthy individuals suggesting that the gradual $\beta$-cell destruction in LADA is insufficient to cause significant weight loss. ${ }^{53}$ An earlier study observed that LADA patients with lower BMI show a more aggressive disease, which can lead to earlier $\beta$-cell exhaustion. ${ }^{54}$ In accordance with this study, elderly LADA patients with lower BMI displayed a lower fasting and 2-hour postprandial C-peptide levels, ${ }^{42}$ indicating that elderly LADA patients with leaner body have poorer residual $\beta$-cell function than those who are obese. On the other hand, data from two large population-based studies showed that overweight and obesity are associated with an increased risk of LADA and that the risk is highest in individuals with a combination of overweight and family history of diabetes. ${ }^{52}$ The authors further suggested that the association with obesity appeared strongest in LADA with low GADA, and that LADA in 31-56\% of individuals could be attributed to overweight/obesity, compared with $70-82 \%$ of all those with Type $2 \mathrm{DM}^{52}$

The observed weight loss with high blood glucose levels might mean a patient is not getting the right amount of insulin or that the loss of glucose through the urine leads to losing calories as well as dehydration, thus leading to rapid weight loss. Moreover, poorly controlled diabetes can cause weight loss through muscle wasting, whereby insulin deficiency found in Type $2 \mathrm{DM}$ both decreases muscle synthesis and increases its breakdown. ${ }^{55}$ Alternatively, patients with LADA were reported to express higher dipeptidyl peptidase 4 (DPP 4) activity than those with Type 1 and Type $2 \mathrm{DM}^{56}{ }^{56}$ whereby its direct addition to skeletal muscle cells has been shown to inhibit insulin signaling, ${ }^{57}$ suggesting that high DPP 4 activity can suppress protein synthesis in skeletal muscle. Moreover, LADA patients were reported to be at high risk 
for the progressive loss of muscle mass and function compared to Type 2 DM. $^{58}$

In our study, TG, cholesterol and HDL-c were nonsignificantly lower in LADA with respect to Type 2 DM, which is in agreement with several studies, ${ }^{28,35,51,59}$ and in line with other studies reporting significant decrease in TG and cholesterol in LADA. ${ }^{5,60}$ The slight decrease in TG might be attributed to the observed associated decrease in insulin resistance (HOMA-IR). Moreover, HDL-c was reported to decrease with age in LADA patient indicating that LADA are at a higher risk of developing cardiovascular complications than Type 2 DM. $^{61}$ Although much evidence has shown LADA patients to have lower BMI, waist-hip ratio, total cholesterol, and TG levels than those with Type 2 DM, some researchers have found that LADA and Type 2 DM were not distinct disease entities beyond the differences explained by BMI. ${ }^{62}$

Overall, our study showed MetS to be significantly lower in LADA with respect to Type $2 \mathrm{DM}$, which is in agreement with previous studies. ${ }^{1,31,60-65}$ The lower MetS in our LADA patients is in concordance with the observed lower risk factors (BMI, TG and BP), and may be attributed to the lower HOMA-IR. A recent study suggested that insulin resistance may account for the residual MetS risk in LADA patients and support the use of insulin sensitizers in LADA. ${ }^{65}$ Metabolic syndrome was also reported not to be a characteristic of autoimmune diabetes and be less prevalent in patients with autoimmune diabetes than in control subjects when glucose was excluded as a variable. ${ }^{61}$ However, another study reported that elderly LADA patients had significantly more components of MetS than those with young LADA, ${ }^{42}$ and have similar MetS prevalence as compared with Type 2 DM patients. Consistent with this, up to $59.2 \%$ of elderly LADA patients have MetS, ${ }^{42}$ which was higher than the $41.9 \%$ reported in European LADA patients ${ }^{61}$ and the $31 \%$ reported in a Chinese study. ${ }^{66}$ Analysis of the data stratified by GADA titre showed that more components of MetS were evident in elderly LADA patients with low titre of GADA. $^{42}$ Therefore, the clinical phenotype of LADA patients varies considerably with GADA titre, whereby low-titre GADA patients are phenotypically more similar to those with Type 2 DM. ${ }^{6,16}$

Fasting blood glucose and HbAlc in our LADA patients were significantly higher, which is in agreement with earlier studies, ${ }^{36,50,59,67}$ and in line with other studies reporting higher FBG and HbAlc levels, though nonsignificant, in LADA compared to Type $2 \mathrm{DM}^{33,51}$
Individuals with GADA-positive were also reported to have a tendency towards a higher proportion of individuals with HbA1c above the optimal target range when compared with GADA-negative subjects. ${ }^{33}$ A recent study suggested that the higher 9-year mean HbA1c levels in LADA patients' entirely explains the higher risk of microvascular complications in LADA patients. ${ }^{68}$ The authors further suggested that $\mathrm{HbA} 1 \mathrm{c}$ should be considered as a therapeutic target to prevent microvascular disease in LADA patients and that immediate and sustained implementation of strict glycemic control is essential for LADA patients to reduce their risk of microvascular complications. Previous UKPDS results showed that $1 \%$ reduction in $\mathrm{HbA} 1 \mathrm{c}$ is associated with a $37 \%$ reduced risk of microvascular disease. ${ }^{69}$ In addition, LADA patients were reported to have worse glycemic regulation than Type 2 DM patients, possibly due to the limited production of endogenous insulin. ${ }^{1,19,60,70}$ This may be attributed to the autoimmune destruction of the $\beta$-cells which has been reported to be present at the diagnosis of LADA. ${ }^{71}$

In our study, both insulin and HOMA- $\beta$ were significantly lower in LADA, which is in agreement with earlier studies. ${ }^{1,9,31,35,51,52}$ These findings indicated that residual $\beta$-cell function in LADA patients was worse than in Type $2 \mathrm{DM}$ patients and that the $\beta$-cell secretory function in LADA deteriorate over time at a 3-fold higher rate than in Type $2 \mathrm{DM}^{72}$ A recent study showed that about a quarter of LADA developed $\beta$-cell dysfunction in the first 8 years' duration, and that GADA titer was the most important discriminatory factor related to higher risk of development of $\beta$-cell dysfunction. ${ }^{73}$ However, another recent study suggested that individuals with low levels of GADA and absence of other antibodies do not experience progressive deterioration of $\beta$-cell function over a 21-month timeframe. ${ }^{74}$ The impaired $\beta$-cell function may be attributed to an autoimmune-mediated $\beta$-cell dysfunction with a loss of $\beta$-cell mass, ${ }^{75}$ which is considered as the main pathogenesis of LADA. ${ }^{11}$ Other contributing factors include the persistent and significant hyperglycaemia in LADA patients which increases production of reactive oxygen species that is considered to mediate glucose toxicity of $\beta$-cell and induce $\beta$-cell apoptosis. ${ }^{76}$ This abnormal and irreversible pancreatic $\beta$-cell damage caused by prolonged exposure to supraphysiological glucose results in decreased insulin gene expression and decreased insulin synthesis and secretion. ${ }^{76}$ Moreover, a number of studies have shown that LADA patients tend to have a more pronounced loss of C-peptide and an increased likelihood 
of insulin treatment compared with Type 2 DM patients. 5,14,17,59,77 Recently, the decline pattern of C-peptide in LADA patients was demonstrated to be biphasic with an initial rapid linear progression followed by a stable mode. ${ }^{73}$

The lower HOMA- $\beta$ in our LADA patients with respect to previous studies conducted in other LADA populations $^{36,68}$ might be explained in view of the fact that our Type $2 \mathrm{DM}$ patients were diagnosed more than 2 months and less than 5 years and therefore their $\beta$-cell mass is much affected (12\%); whereas those of other populations the participants were newly diagnosed Type $2 \mathrm{DM}$ in which their $\beta$-cell mass was still preserved and not much affected $(\approx 40 \%)$. In addition, almost all our LADA patients were not on insulin treatment. Consistent data from randomized clinical trials showed the importance of an early initiation of insulin therapy in LADA avoiding the use of secretagogues like sulphonylureas. ${ }^{78}$ Generally, a higher proportion of GADA-positive patients were receiving insulin therapy when compared with those who were GADA-negative. ${ }^{33}$ However, in our study only two out of the twelve GAD-positive (16.7\%) were on insulin treatment, which is in line with several studies reporting $15 \%, 18 \%$ and $37 \%$ of their LADA patients were on insulin at the time of the study. ${ }^{34,77,79}$

Insulin resistant (HOMA-IR) was non-significant lower in our LADA patients by $16.7 \%$, which is in agreement with various studies from Asia and Western countries reporting that LADA is being characterized by insulin resistance, but to a lesser extent than Type 2 $\mathrm{DM} ;{ }^{4,51,52,62,80,81}$ which has been attributed to differences in BMI. ${ }^{62,80}$ The non-significant decrease observed in our LADA patients is likely to be attributed to the lower power (only 12 LADA) and not because of the absence of difference between the two studied groups. Thus, confirming that LADA patients still share insulin resistance with Type 2 DM but display a more severe defect in $\beta$-cell function. ${ }^{82}$ In contrast, a previous study showed insulin resistance to be significant higher in LADA patients. ${ }^{28}$ This discrepancy with our results might be due to differences in treatment profile of subjects whereby their patients were already on insulin therapy whereas our patients were mostly on oral hypoglycaemic agents. Recently, elderly LADA patients were reported to display higher insulin resistance than those with young LADA patients but were not significant different from that in Type $2 \mathrm{DM}$ patients. ${ }^{42}$ Moreover, the contribution of insulin resistance to the development of LADA was shown to depend on the degree of autoimmunity, whereby LADA patients with less autoimmune activity as indicated by low GADA levels tend to be more insulin resistant than those with high GADA levels. $^{52}$

To the best of our knowledge, this is the first study to evaluate patients with LADA in Yemen. However, our study has some limitations. First, this was a hospitalbased study, and selection of the study population may have had some bias. Second, the median disease duration was 0.9 year in GADA-positive (LADA) patients, while it was longer (2 years) in GADA-negative (Type 2 DM) patients. Third, since only GADA-positivity was considered for the diagnosis of LADA we cannot exclude the possibility that analysis of other antibodies (eg, islet antigen-2 antibody, insulin autoantibody, and zinc transporter 8) could contribute to the overall prevalence, particularly among those with GADA-negative. Fourth, the incidence and clinical features of autoimmune diabetes may vary between regions and ethnicities. Fifth, due to the small sample size of the LADA group, the results of the current study cannot be generalized and because of this lower power the absence of significant differences in some variables (eg, HOMA-IR) between LADA and Type 2 DM may not be because of the absence of differences between the two groups.

\section{Conclusion}

The results showed the prevalence of LADA based on the positivity of glutamic acid decarboxylase autoantibodies in Yemeni Type $2 \mathrm{DM}$ is lower than many of those reported in the literature with no gender preference. Compared with GADA-negative Type 2 DM, LADA patients had earlier onset of diabetes and lower prevalence of metabolic syndrome. LADA patients share insulin resistance with Type 2 DM but display a severe defect in $\beta$-cell function. The results highlight the importance of an early diagnosis of LADA, which would avoid patients receiving unnecessary oral medication, allowing safe and effective insulin therapies as well as helping to predict the disease course and reducing long-term diabetes complications. Further epidemiological and clinical studies with larger sample sizes will demonstrate the prevalence and increase awareness of LADA.

\section{Abbreviations}

$\mathrm{BMI}$, body mass index; $\mathrm{BP}$, blood pressure; DBP, diastolic blood pressure; ECL, electrochemiluminescence immunoassay; ELISA, enzyme linked immunoassay; FBG, fasting blood glucose; GADA, glutamic acid decarboxylase antibody; 
HbA1c, glycated hemoglobin; HDL-c, high density lipoprotein cholesterol; HOMA, homeostasis model assessment; HOMA-IR, insulin resistance; HOMA- $\beta$, $\beta$-cell function; LADA, latent autoimmune diabetes in adults; LDL-c, low density lipoprotein cholesterol; MetS, metabolic syndrome; SD, standard deviation; SPSS, Statistically Package for Social Sciences; SBP, systolic blood pressure; TG, triglycerides; WC, waist circumference.

\section{Data Sharing Statement}

The data set generated and/or analyzed during this study are included in this submitted manuscript and is available from the corresponding author on reasonable request.

\section{Ethical Approval}

The study was approved by the Institutional Ethical Committee, Faculty of Medicine and Health Sciences, Sana'a University. The study was in compliance with the Declaration of Helsinki for clinical research. All the patients provided written informed consent before participating in the study.

\section{Consent to Publish}

All authors approved the submitted manuscript.

\section{Acknowledgments}

The authors thank all the study participants.

\section{Disclosure}

All authors report no conflicts of interest for this work.

\section{References}

1. Carlsson S. Etiology and pathogenesis of latent autoimmune diabetes in adults (LADA) compared to type 2 diabetes. Front Physiol. 2019;10:Article 320. doi:10.3389/fphys.2019.00320

2. Luo SM, Liu BW, Yin WF, Li X, Zhou ZG. A long and winding road to understand latent autoimmune diabetes in adults. Chin Med J. 2021;134:1-4. doi:10.1097/CM9.0000000000001321

3. Buzzetti R, Zampetti S, Maddaloni E. Adult-onset autoimmune diabetes: current knowledge and implications for management. Nat Rev Endocrinol. 2017;13(11):674-686. doi:10.1038/nrendo.2017.99

4. Tuomi T, Santoro N, Caprio S, Cai M, Weng J, Groop L. The many faces of diabetes: a disease with increasing heterogeneity. Lancet. 2014;383:1084-1094. doi:10.1016/S0140-6736(13)62219-9

5. Hawa MI, Kolb H, Schloot N, et al. Adult-onset autoimmune diabetes in Europe is prevalent with a broad clinical phenotype: action LADA 7. Diabetes Care. 2013;36:908-913. doi:10.2337/dc12-0931

6. Laugesen E, Ostergaard JA, Leslie RD. Latent autoimmune diabetes of the adult: current knowledge and uncertainty. Diabetic Med. 2015;32 (7):843-852. doi: 10.1111/dme.12700

7. Leslie RD, Williams R, Pozzilli P. Clinical review: type 1 diabetes and latent autoimmune diabetes in adults: one end of the rainbow. J Clin Endocrinol Metab. 2006;91:1654-1659. doi:10.1210/jc.2005-1623
8. Howson JM, Rosinger S, Smyth DJ, Boehm BO, Todd JA. Genetic analysis of adult-onset autoimmune diabetes. Diabetes. 2011;60:2645-2653. doi:10.2337/db11-0364

9. Hernandez M, Mollo A, Marsal JR, et al. Insulin secretion in patients with latent autoimmune diabetes (LADA): half way between type 1 and type 2 diabetes. Action LADA 9. BMC Endocr Disord. 2015;15: Article no. 1. doi:10.1186/1472-6823-15-1

10. Naik RG, Brooks-Worrell BM, Palmer JP. Latent autoimmune diabetes in adults. $J$ Clin Endocrinol Metab. 2009;94:4635-4644. doi:10.1210/jc.2009-1120

11. Fourlanos S, Dotta F, Greenbaum CJ, et al. Latent autoimmune diabetes in adults (LADA) should be less latent. Diabetologia. 2005;48:2206-2212. doi:10.1007/s00125-005-1960-7

12. Maddaloni E, Lessan N, Al Tikriti A, Buzzetti R, Pozzilli P, Barakat MT. Latent autoimmune diabetes in adults in the United Arab Emirates: clinical features and factors related to insulin-requirement. PLoS One. 2015;10(8):e0131837. doi:10.1371/ journal.pone. 0131837

13. Xiang Y, Zhou Z, Deng C, Leslie RD. Latent autoimmune diabetes in adults in Asians: similarities and differences between east and west. J Diabetes. 2013;5(2):118-126. doi:10.1111/1753-0407.12029

14. Zhou Z, Xiang Y, Ji L, et al. Frequency, immunogenetics, and clinical characteristics of latent autoimmune diabetes in China (LADA China Study): a nationwide, multicenter, clinic-based cross-sectional study. Diabetes. 2013;62:543-550. doi:10.2337/db12-0207

15. Maddaloni E, Pastore G, Del Buono MG, et al. High prevalence of autoimmune diabetes and poor Glycaemic control among adults in Madagascar: a brief report from a humanitarian health campaign in Ambanja. J Diabetes Res. 2017;2017:1-5. doi:10.1155/2017/3860674.

16. Buzzetti R, Di PS, Giaccari A, et al. High titer of autoantibodies to GAD identifies a specific phenotype of adult-onset autoimmune diabetes. Diabetes Care. 2007;30:932-938. doi:10.2337/dc06-1696

17. Radtke MA, Midthjell K, Nilsen TI, Grill V. Heterogeneity of patients with latent autoimmune diabetes in adults: linkage to autoimmunity is apparent only in those with perceived need for insulin treatment: results from the Nord-Trondelag Health (HUNT) Study. Diabetes Care. 2009;32(2):245-250. doi:10.2337/dc08-1468

18. Qi X, Sun J, Wang J, et al. Prevalence and correlates of latent autoimmune diabetes in adults in Tianjin, China. Diabetes Care. 2011;34:66-70. doi:10.2337/dc10-0488

19. Kumar A, de Leiva A. Latent autoimmune diabetes in adults (LADA) in Asian and European populations. Diabetes/ Metab Res Rev. 2017;33(5):e2890. doi:10.1002/dmrr.2890

20. Canivell S, Gomis R. Diagnosis and classification of autoimmune diabetes mellitus. Autoimmun Rev. 2014;13(4-5):403-407. doi:10.1016/j.autrev.2014.01.020

21. Maioli M, Pes GM, Delitala G, et al. Number of autoantibodies and HLA genotype, more than high titers of glutamic acid decarboxylase autoantibodies, predict insulin dependence in latent autoimmune diabetes of adults. Eur J Endocrinol. 2010;163:541-549. doi:10.1530/ EJE-10-0427

22. Thomas NJ, Jones SE, Weedon MN, Shields BM, Oram RA, Hattersley AT. Frequency and phenotype of type 1 diabetes in the first six decades of life: a cross-sectional, genetically stratified survival analysis from UK Biobank. Lancet Diabetes Endocrinol. 2018;6 (2):122-129. doi:10.1016/S2213-8587(17)30362-5

23. Leslie RD, Kolb H, Schloot NC, et al. Diabetes classification: grey zones, sound and smoke: action LADA 1. Diabetes Metab Res Rev. 2008;24(7):511-519. doi:10.1002/dmrr.877

24. Alberti KGMM, Eckel RH, Grundy SM, et al. Harmonizing the metabolic syndrome: a joint interim statement of the International Diabetes Federation Task Force on Epidemiology and Prevention; National Heart, Lung, and Blood Institute; American Heart Association; World Heart Federation; International Atherosclerosis Society; and International Association for the Study of Obesity. Circulation. 2009;120:1640-1645. 
25. Park Y, Hong S, Park L, Woo J, Baik S, Nam M. LADA prevalence estimation and insulin dependency during follow-up. Diabetes Metab Res Rev. 2011;27:975-979. doi:10.1002/dmrr.1278

26. Zinman B, Kahn SE, Haffner SM, O’Neill MC, Heise MA, Freed MI Phenotypic characteristics of GAD antibody-positive recently diagnosed patients with type 2 diabetes in North America and Europe. Diabetes. 2004;53:3193-3200. doi:10.2337/diabetes.53.12.3193

27. Panz V, Kalk W, Zonvanis M, Joffe B. Distribution of autoantibodies to glutamic acid decarboxylase across the spectrum of diabetes mellitus seen in South Africa. Diabetes Med. 2000;7:524-527. doi:10.1046/j.1464-5491.2000.00324.x

28. Salem SD, Saif-Ali R, Muniandy S, Al-Hamodi Z, Ismail IS. Comparison of adults with insulin resistance (IR) in latent autoimmune diabetes versus IR in glutamic acid decarboxylase antibody-negative. Ann Acad Med Singapore. 2014;43:107-112.

29. Maioli M, Alejandro E, Tonolo G, Gilliam L, Bekris L, Hampe C. Epitope-restricted 65-kilodalton glutamic acid decarboxylase autoantibodies among new-onset Sardinian type 2 diabetes patients define phenotypes of autoimmune diabetes. J Clin Endocrinol Metab. 2004;89:5675-5682. doi:10.1210/jc.2004-0864

30. Lutale JK, Thordarson H, Holm P, Eide GE, Vetvik K. Islet cell autoantibodies in African patients with type 1 and type 2 diabetes in Dar el Salaam Tanzania: a cross-sectional study. J Autoimmune Dis. 2007;4. doi:10.1186/1740-2557-4-4

31. Katulanda P, Shine B, Katulanda W, et al. Diabetes mellitus among young adults in Sri Lanka: role of GAD antibodies in classification and treatment: the Sri Lanka Young Diabetes study. Diabetologia. 2008;51:1368-1374. doi:10.1007/s00125-008-1052-6

32. Kanungo A, Sanjeevi CB. IA-2 autoantibodies are predominant in latent autoimmune diabetes in adults patients from eastern India. Ann NY Acad Sci. 2003;1005:390-394. doi:10.1196/annals.1288.065

33. Ipadeola A, Adeleyea JO, Akinladeba KS. Latent autoimmune diabetes amongst adults with type 2 diabetes in a Nigerian tertiary hospital. Prim Care Diabetes. 2015;9:231-236. doi:10.1016/j.pcd.2014.09.003

34. Adeleye O, Ogbera A, Fasanmade O, et al. Latent autoimmune diabetes mellitus in adults (LADA) and it's characteristics in a subset of Nigerians initially managed for type 2 diabetes. Int Arch Med. 2012;5:23. doi:10.1186/1755-7682-5-23.

35. Agyei-Frempong MT, Titty FV, Owiredu WK, Eghan BA. The prevalence of autoimmune diabetes among diabetes mellitus patient in Kumasi, Ghana. Pakistan J Biol Sci. 2008;11(19):2320-2325. doi:10.3923/pjbs.2008.2320.2325

36. Maddaloni E, Coleman RL, Pozzilli P, Holman RR. Long-term risk of cardiovascular disease in individuals with latent autoimmune diabetes in adults (UKPDS 85). Diabetes Obes Metab. 2019;21(9):2115-2122. doi:10.1111/dom. 13788

37. Tuomi T, Carlsson A, Li H, et al. Clinical and genetic characteristics of type 2 diabetes with and without GAD antibodies. Diabetes. 1999;48:150-157. doi:10.2337/diabetes.48.1.150

38. Davis TM, Zimmet P, Davis WA, Bruce DG, Fida S, Mackay IR. Autoantibodies to glutamic acid decarboxylase in diabetic patients from a multi-ethnic Australian community: the Fremantle Diabetes Study. Diabetic Med. 2000;17:667-674. doi:10.1046/j.14645491.2000.00359.x

39. Soriguer-Escofet F, Esteva I, Rojo-Martinez G, Adana SR, Catalá M, José Merelo M. Prevalence of latent autoimmune diabetes of adults (LADA) in Southern Spain. Diabetes Res Clin Pract. 2002;56:213-220. doi:10.1016/S0168-8227(02)00002-5

40. Fourlanos S, Perry C, Stein MS, Stankovich J, Harrison LC, Colman PG. A clinical screening tool identifies autoimmune diabetes in adults. Diabetes Care. 2006;29:970-975. doi:10.2337/dc05-2101

41. Sorgjerd EP, Skorpen F, Kvaloy K, Midthjell K, Grill V. Time dynamics of autoantibodies are coupled to phenotypes and add to the heterogeneity of autoimmune diabetes in adults: the HUNT study, Norway. Diabetologia. 2012;55:1310-1318. doi:10.1007/s00125012-2463-y
42. Niu X, Luo S, Li X, et al. Identification of a distinct phenotype of elderly latent autoimmune diabetes in adults: LADA China Study 8. Diabetes Metab Res Rev. 2019;35:e3068. doi:10.1002/dmrr.3068

43. Hamaguchi K, Kimura A, Kusuda Y, Yamashita T, Yasunami M, Takahasi M. Clinical and genetic characteristics of GAD-antibody positive patients initially diagnosed as having type 2 diabetes. Diabetes Res Clin Pract. 2004;66:163-171. doi:10.1016/j. diabres.2004.02.019

44. Kim nam J, Park J, Kang E, et al. Clinical and biochemical characteristics of non-obese type 2 diabetic patients with glutamic acid decarboxylase antibody in Korea. Metab Clin Exp. 2006;55:1107-1112. doi:10.1016/j.metabol.2006.04.006

45. Elder DA, Herbers PM, Weis T, Standiford D, Woo JG, D'Alessio DA. $\beta$-cell dysfunction in adolescents and adults with newly diagnosed type 2 diabetes. J Pediatr. 2012;160(6):904-910. doi:10.1016/j.jpeds.2011.12.002

46. Horton V, Stratton I, Bottazzo GF, et al. Genetic heterogeneity of autoimmune diabetes: age of presentation in adults is influenced by HLA DRB1 and DQB1 genotypes (UKPDS 43). Diabetologia. 1999;42(5):608-616. doi:10.1007/s001250051202

47. Bakhtadze E, Cervin C, Lindholme E, et al. Common variants in the TCF7L2 gene help to differentiate autoimmune from nonautoimmune diabetes in young (15-34 years) but not in middleaged (40-59 years) diabetic patients. Diabetologia. 2008;51 (12):2224-2232. doi:10.1007/s00125-008-1161-2

48. Paschke A, Grzelka A, Zawada A, Zozulińska-Ziołkiewicz D. Clinical characteristics and autoantibody pattern in newly diagnosed adult-onset autoimmune diabetes. Pol Arch Med Wewn. 2013;123(78):401-408.

49. Birkeland KI, Grill V, Wium C, et al. The association of basal insulin treatment versus standard care with outcomes in anti-GAD positive and negative subjects: a post-hoc analysis of the ORIGIN trial. Diabetes Obes Metab. 2019;21:429-433. doi:10.1111/dom.13528

50. Guglielmi C, Palermo A, Pozzilli P. Latent autoimmune diabetes in the adults (LADA) in Asia: from pathogenesis and epidemiology to therapy. Diabetes Metab Res Rev. 2012;28:40-46. doi:10.1002/ dmrr.2345

51. Szepietowska B, Głębocka A, Puch U, Górska M, Szelachowska M. Latent autoimmune diabetes in adults in a population-based cohort of Polish patients with newly diagnosed diabetes mellitus. Arch Med Sci. 2012;8(3):491-495. doi:10.5114/aoms.2012.29527

52. Hjort R, Ahlqvist E, Carlsson PO, et al. Overweight, obesity and the risk of LADA: results from a Swedish case-control study and the Norwegian HUNT Study. Diabetologia. 2018;61:1333-1343. doi:10.1007/s00125-018-4596-0

53. Brahmkshatriya PP, Mehta AA, Saboo BD, Goyal RK. Characteristics and prevalence of latent autoimmune diabetes in adults (LADA). ISRN Pharmacol. 2012;2012(Article):580202. doi:10.5402/2012/580202

54. Lohmann T, Kellner K, Verlohren HJ, et al. Titre and combination of ICA and autoantibodies to glutamic acid decarboxylase discriminate two clinically distinct types of latent autoimmune diabetes in adults (LADA). Diabetologia. 2001;44:1005-1010. doi:10.1007/ s001250100602

55. Rosenbaum M, Sy M, Pavlovich K, Leibel RL, Hirsch J. Leptin reverses weight loss-induced changes in regional neural activity responses to visual food stimuli. J Clin Invest. 2008;118 (7):2583-2591.

56. Duvnjak L, Blaslov K, Vučić Lovrenčić M, Knežević Ćuća J. Persons with latent autoimmune diabetes in adults express higher dipeptidyl peptidase-4 activity compared to persons with type 2 and type 1 diabetes. Diabetes Res Clin Pract. 2016;121:119-126. doi:10.1016/ j.diabres.2016.09.013

57. Lamers D, Famulla S, Wronkowitz N, et al. Dipeptidyl peptidase 4 is a novel adipokine potentially linking obesity to the metabolic syndrome. Diabetes. 2011;60(7):1917-1925. doi:10.2337/db10-1707 
58. Bouchi R, Fukuda T, Takeuchi T, et al. Association of sarcopenia with both latent autoimmune diabetes in adults and type 2 diabetes: a cross-sectional study. $J$ Diabetes Complications. 2017;31 (6):992-996. doi:10.1016/j.jdiacomp.2017.02.021

59. Tam AA, Ozdemir D, Bestepe N, et al. Low rate of latent autoimmune diabetes in adults (LADA) in patients followed for type 2 diabetes: a single center's experience in Turkey. Arch Endocrinol Metab. 2020;64(5):584-590.

60. Olsson L, Grill V, Midthjell K, Ahlbom A, Andersson T, Carlsson S. Mortality in adult-onset autoimmune diabetes is associated with poor glycemic control. Diabetes Care. 2013;36:3971-3978. doi:10.2337/ dc13-0564

61. Hawa MT, Thivolet C, Mauricio D, et al. Metabolic syndrome and autoimmune diabetes: action LADA 3. Diabetes Care. 2009;32:160-164. doi:10.2337/dc08-1419

62. Juhl CB, Bradley U, Holst JJ, et al. Similar weight-adjusted insulin secretion and insulin sensitivity in short-duration late autoimmune diabetes of adulthood (LADA) and type 2 diabetes: action LADA 8. Diabetic Med. 2014;31(8):941-945. doi:10.1111/dme.12434

63. Roh MO, Jung CH, Kim BY, Mok JO, Kim CH. The prevalence and characteristics of latent autoimmune diabetes in adults (LADA) and its relation with chronic complications in a clinical department of a university hospital in Korea. Acta Diabetol. 2013;50:129-134. doi:10.1007/s00592-010-0228-y

64. Wod M, Yderstræde KB, Halekoh U, Beck-Nielsen H, Højlund K. Metabolic risk profiles in diabetes stratified according to age at onset, islet autoimmunity and fasting C-peptide. Diabetes Res Clin Pract. 2017;134:62-71. doi:10.1016/j.diabres.2017.09.014

65. Li X, Cao C, Tang X, et al. Prevalence of metabolic syndrome and its determinants in newly-diagnosed adult-onset diabetes in China: a multi-center, cross-sectional survey. Front Endocrinol. 2019;10:661. doi: $10.3389 /$ fendo.2019.00661

66. Lu J, Hou X, Zhang L, et al. Associations between clinical characteristics and chronic complications in latent autoimmune diabetes in adults and type 2 diabetes. Diabetes/ Metab Res Rev. 2015;31 (4):411-420. doi:10.1002/dmrr.2626

67. Sia H-K, Tu S-T, Liao P-Y, Lin K-H, Kor C-T, Yeh -L-L. A convenient diagnostic tool for discriminating adult-onset glutamic acid decarboxylase antibody-positive autoimmune diabetes from type 2 diabetes: a retrospective study. Peer J. 2020;8:e8610. doi:10.7717/ peerj. 8610

68. Maddaloni E, Coleman RL, Agbaje O, Buzzetti R, Holman RR. Time-varying risk of microvascular complications in latent autoimmune diabetes of adulthood compared with type 2 diabetes in adults: a post-hoc analysis of the UK Prospective Diabetes Study 30-year follow-up data (UKPDS 86). Lancet Diabetes Endocrinol. 2020;8 (3):206-215. doi:10.1016/S2213-8587(20)30003-6

69. Stratton IM, Adler AI, Neil HA, et al. Association of glycaemia with macrovascular and microvascular complications of type 2 diabetes (UKPDS 35): prospective observational study. BMJ. 2000;321:405-412. doi:10.1136/bmj.321.7258.405
70. Andersen CD, Bennet L, Nyström L, et al. Worse glycaemic control in LADA patients than in those with type 2 diabetes, despite a longer time on insulin therapy. Diabetologia. 2013;56(2):252-258. doi:10.1007/s00125-012-2759-y

71. Stenström G, Gottsäter A, Bakhtadze E, Berger B, Sundkvist G. Latent autoimmune diabetes in adults: definition, prevalence, $\beta$-cell function and treatment. Diabetes. 2005;54:S68-72. doi:10.2337/diabetes.54.suppl_2.S68

72. Yang L, Zhou ZG, Huang G, Ouyang LL, Li X, Yan X. Six-year follow up of pancreatic beta cell function in adults with latent autoimmune diabetes. World J Gastroenterol. 2005;11:2900-2905. doi:10.3748/wjg.v11.i19.2900

73. Li X, Chen Y, Xie Y, Xiang Y, Yan X, Huang G. Decline pattern of beta-cell function in adult-onset latent autoimmune diabetes: an 8-year prospective study. J Clin Endocrinol Metab. 2020;105 (7):2331-2340. doi:10.1210/clinem/dgaa205

74. Hals IK, Fleiner HF, Reimers N, et al. Investigating optimal $\beta$-cellpreserving treatment in latent autoimmune diabetes in adults: results from a 21-month randomized trial. Diabetes Obes Metab. 2019;21 (10):2219-2227. doi:10.1111/dom.13797

75. Subauste A, Gianani R, Chang AM, et al. Islet autoimmunity identifies a unique pattern of impaired pancreatic beta-cell function, markedly reduced pancreatic beta cell mass and insulin resistance in clinically diagnosed type 2 diabetes. PLoS One. 2014;9(9):e106537. doi:10.1371/journal.pone.0106537

76. Robertson RP. Chronic oxidative stress as a central mechanism for glucose toxicity in pancreatic islet beta cells in diabetes. J Biol Chem. 2004;279:42351-42354. doi:10.1074/jbc.R400019200

77. Hwangbo Y, Kim JT, Kim EK, et al. Prevalence and clinical characteristics of recently diagnosed type 2 diabetes patients with positive anti-glutamic acid decarboxylase antibody. Diabetes Metab J. 2012;36(2):136-143. doi:10.4093/dmj.2012.36.2.136

78. Maruyama T, Tanaka S, Shimada A, et al. Insulin intervention in slowly progressive insulin-dependent (type 1) diabetes mellitus. J Clin Endocrinol Metab. 2008;93:2115-2121. doi:10.1210/jc.2007-2267

79. Pan CY, So WY, Khalid BA, et al. Metabolic, immunological and clinical characteristics in newly diagnosed Asian diabetes patients aged 12-40 years. Diabetic Med. 2004;21(9):1007-1013. doi:10.1111/j.1464-5491.2004.01287.x

80. Chiu HK, Tsai EC, Juneja R, et al. Equivalent insulin resistance in latent autoimmune diabetes in adults (LADA) and type 2 diabetic patients. Diabetes Res Clin Pract. 2007;77:237-244. doi:10.1016/j. diabres.2006.12.013

81. Zaharia OP, Bobrov P, Strassburger K, et al. Metabolic characteristics of recently diagnosed adult-onset autoimmune diabetes mellitus. J Clin Endocrinol Metab. 2018;103(2):429-437. doi:10.1210/ jc. 2017-01706

82. Carlsson A, Sundkvist G, Groop L, Tuomi T. Insulin and glucagon secretion in patients with slowly progressing autoimmune diabetes (LADA). J Clin Endocinol Metab. 2000;85(1):76-80.

\section{Publish your work in this journal}

Diabetes, Metabolic Syndrome and Obesity: Targets and Therapy is an international, peer-reviewed open-access journal committed to the rapid publication of the latest laboratory and clinical findings in the fields of diabetes, metabolic syndrome and obesity research. Original research, review, case reports, hypothesis formation, expert opinion and commentaries are all considered for publication. The manuscript management system is completely online and includes a very quick and fair peer-review system, which is all easy to use. Visit http://www.dovepress.com/testimonials.php to read real quotes from published authors. 